\title{
"What is the impact of utilizing so-called best practices in percutaneous coronary intervention?": an interview with Atul Gupta
}

\author{
Atul Gupta*,1 \\ ${ }^{1}$ Royal Philips, Amstelplein 2, 1096 BC Amsterdam, The Netherlands \\ * Author for correspondence: atul.gupta@philips.com
}

\begin{abstract}
Atul Gupta, MD* speaks to Adam Price-Evans, Managing Commissioning Editor of Future Cardiology. Atul Gupta is the Global Chief Medical Officer for the business group Image Guided Therapy at Philips, providing medical guidance to Philips' clinical vision and strategy. As a practicing interventional and diagnostic radiologist, he also serves as a key external clinical voice for Image Guided Therapy. His key responsibilities include supporting innovation and product development in cardiology, peripheral vascular, surgical, oncology interventions, clinical education, office-based labs, medical affairs and new business development and ventures. He went to medical school and completed his postgraduate training in diagnostic radiology and a fellowship in interventional radiology. He maintains a clinical practice, performing interventional and diagnostic radiology in both hospital and office-based lab settings.
\end{abstract}

First draft submitted: 6 September 2017; Accepted for publication: 11 January 2018; Published online: 23 January 2018

Keywords: 3-vessel disease $\bullet$ iFR • instantaneous wave-free ratio • intravascular ultrasound • IVUS • PCI • percutaneous coronary intervention • second-generation drug-eluting stents • SYNTAX II

Could you begin by introducing yourself, your background \& telling us a bit about your work with Philips to date?

My name is Atul Gupta and I am a practicing physician with over 20 years of experience in minimally invasive interventional procedures. I went to medical school in New York (USA) and came to Philadelphia for my advanced training in diagnostic radiology and interventional radiology, both at the University of Pennsylvania (PA, USA). I have practiced here in the Philadelphia region for 17 years and throughout that time, I have collaborated with Philips on various research projects largely related to 3D navigation and image-guidance procedures. I formally joined Philips a few years ago as the Chief Medical Officer for the Philips Image Guided Therapy (IGT) business. My role is to provide medical leadership and clinical support to all of our global IGT businesses including interventional systems (fixed and mobile) and medical devices. I am actively involved with product development, new business development, clinical education and, in general, supporting the Philips vision, which is to improve the lives of three billion people a year by 2025. On a personal level, I think what attracted me to Philips is the opportunity to influence patient care on a far greater level than I could as a single person in private practice. We actually crunched the numbers and it turns out that the IGT solutions in Philips are used to treat one patient every single second somewhere on this planet. To me, that was an extraordinarily compelling and amazing opportunity.

Philips technology was used as part of the SYNTAX II trial, which investigated modern developments in percutaneous coronary intervention for 3-vessel disease since the SYNTAX I trial. Could you tell us a bit more about the study rationale $\&$ its primary goals and objectives?

The rationale was to answer the question, 'what is the impact of utilizing so-called best practices in percutaneous coronary intervention (PCI)'? The original SYNTAX I trial suggested that for patients with 3-vessel disease (3VD), coronary artery bypass graft $(\mathrm{CABG})$ was the preferred strategy for revascularization. However, that trial was performed over 10 years ago and looked at patients from 2005 to 2007. Since then, there have been major

Future $\because \cdots$ Medicine 
technologic and procedural developments in PCI. The SYNTAX II researchers therefore felt that by using newer best practices in PCI, we can further improve outcomes in patients with $3 \mathrm{VD}$. The best practices specifically assessed included physiology guidance with instantaneous wave-free ratio (iFR), second-generation drug-eluting stents, intravascular ultrasound (IVUS) to guide stent implantations and the SYNTAX II score, a clinical tool combining anatomic and clinical factors to reduce variability. The study also looked at new chronic total occlusion revascularization techniques and guideline-directed medical therapy. Incorporating these best practices formed the SYNTAX II strategy. The primary end point was a composite of major adverse cardiovascular and cerebrovascular events (MACCE) at 1 year and this was compared with matched patients treated with PCI in the original SYNTAX I trial.

1-year data from the SYNTAX II trial were presented at the ESC Congress 2017 in Barcelona (Spain). Could you describe the trial design \& the results presented?

The SYNTAX II trial was a multicenter, open-label and single-arm study that investigated the impact of newer PCI techniques on clinical outcomes in 3VD. The trial looked at 454 patients at 22 centers across four European countries. I think the results were rather compelling. The 1-year results in SYNTAX II showed a statistically significant lower rate of MACCE for the SYNTAX II patients versus the SYNTAX I PCI patients (10.6 vs 17.4\%, respectively; $\mathrm{p}=0.006$ ). The SYNTAX II strategy also yielded a lower rate of myocardial infarction (1.4 vs $4.8 \%$, respectively; $\mathrm{p}=0.007)$, significantly lower rates of repeat revascularization ( $8.2 \mathrm{vs} 13.7 \%$, respectively; $\mathrm{p}=0.0015)$ and stent thrombosis $(0.7$ vs $2.7 \%$, respectively; $\mathrm{p}=0.045)$. While not the aim of the study, the researchers also attempted to perform an initial exploratory analysis with the historical CABG cohort of the original SYNTAX I trial. At 1 year, the MACCE rate for the SYNTAX I CABG patients was noninferior to SYNTAX II PCI patients (11.2 vs $10.6 \%$, respectively).

\section{What do you think are the main implications of these results \& how do you think they will impact clinical practice for 3VD in the future?}

I believe the real world implication of the SYNTAX II study is that angiography alone is not enough to determine the treatment pathway for complex PCI. A state-of-the-art PCI technique, which includes iFR and IVUS, can contribute to improved patient outcomes. The use of iFR may also lead to fewer stents being used, which could have a cost implication. If you incorporate all of the modern tools together to optimize PCI, we can improve the outcome. Again, this means using physiology or iFR to guide the intervention, as well as imaging such as IVUS to optimize stent deployment. This study gives confidence to interventional cardiologists currently using these modern PCI techniques and to cardiologists waiting for clinical validation before using this technology.

\section{What do you think will be the next stages in this research \& are there any barriers to overcome?}

Since this is a historical comparison rather than a randomized trial, we must be careful not to overinterpret or overanalyze the results. Despite this, I believe this research shows that integrating technologic developments such as iFR and IVUS in PCI can help achieve better outcomes. The logical next step would be a new trial that compares today's modern PCI, using all of the best practices included in SYNTAX II, with CABG in 3VD. In today's cost-conscious healthcare environment, it would also be interesting to assess if we are not only improving patient outcomes but if we are also improving cost. Interestingly, SYNTAX II found that physiological guidance resulted in fewer lesions being stented. We may therefore be able to treat $3 \mathrm{VD}$ in a minimally invasive, more economical way which improves outcomes, reduces recovery times and highlights which lesions do and do not need treatment.

\section{Philips also showcased a number of cardiological innovations at the ESC Congress 2017. Could you tell us about some of the key products $\&$ how they will result in improvements in cardiovascular care?}

Azurion, Philips' next-generation IGT platform, was front and center at ESC 2017. This interventional suite enables physicians to perform a range of complex interventions, optimize their lab performance and provide superior care. Supporting Azurion is a new suite of tools, including the iFR roadmap, which integrates iFR and angiographic imaging in real-time. This new technology enables physicians to more confidently see and treat coronary disease. It works by automatically overlaying images of the patient's arteries with the correlating physiologic data measurements, an example of uniquely marrying our device to our angiography system. This offers an automated system that 
requires no additional steps or work stations which is important because as we know, if these tools are cumbersome, they are simply not used.

We also introduced the EPIQ with HeartModel at ESC 2017, an advanced ultrasound platform with a new live 3D-transesophageal transducer which assists in both treatment and diagnosis. The ability to fuse live $\mathrm{x}$-ray with live echo ultrasound images offers interventionalists, like me, the best of both worlds. Using Philips' EchoNavigator image fusion, we are now able to precisely navigate the device with the x-ray and still see the soft tissue of the beating heart with the advanced echo.

\section{Philips has recently announced a collaboration agreement with HeartFlow, Inc. (CA, USA) which will involve the promotion of HeartFlow FFRct Analysis in conjunction with Philips' advanced catheters. Could you please tell us more about the aim of this collaboration?}

Today, more than half of the patients that come into a cardiac catheterization lab may actually not need to be there. Unnecessary catheterizations are potentially producing risk for cardiac patients and there is no question that this is also increasing costs. If we can improve noninvasive screening, hopefully it will benefit not just patients but also healthcare systems. At Philips, we believe that collaboration leads to the best innovation. By collaborating with HeartFlow, we aim to enable greater access to physiologic-guided triaging of patients and reduce the cost of care. It is our goal to push the envelope continuously to see how we can further help cardiologists to guide, treat and confirm the right coronary therapy in the interventional suite.

Financial \& competing interests disclosure

A Gupta is an employee of Philips. The interviewee has no other relevant affiliations or financial involvement with any organization or entity with a financial interest in or financial conflict with the subject matter or materials discussed in the manuscript apart from those disclosed. No writing assistance was utilized in the production of this manuscript.

\section{Disclaimer}

The opinions expressed in this interview are those of the interviewee and do not necessarily reflect the views of Future Medicine Ltd. 
